\title{
Construction of Test Measuring Mental Rotation Ability of Adolescent High School Students
}

\author{
Sumona Datta ${ }^{1}$, Debdulal Dutta Roy $^{2}$
}

\section{ABSTRACT}

One important aspect of Visuospatial Reasoning is the ability to rotate an object mentally. Presently, one of the most popular and widely used test of Mental Rotation is the Redrawn version of Vandenberg \& Kuse Mental Rotation test (VMRT) However, this test has often been criticised to be more difficult because of its complicated scoring system. Present study compared this standard test of mental rotation ability with a newly constructed test with less number of items and less complicated scoring system. We collected data from 147 adolescent school students (Mean age=13.10 years; $\mathrm{SD}=1.84$ ) by administering both the tests consecutively. Findings showed that VMRT has very high difficulty index as compared to the newly constructed test. The latter also proved to be a more reliable and valid measure of mental rotation ability as compared to VMRT. Discussion focused on the relative advantage of the newly constructed test for assessing mental rotation ability.

Keywords: Mental Rotation, Cognitive test, Vandenberg \& Kuse Mental Rotation test,

Psychometric test construction.

\section{Mental Rotation Ability}

One of our very important cognitive components is Visuospatial reasoning, often considered to be a component of intelligence (Thurstone, 1938). Visuospatial reasoning (VSR) is the ability to manipulate visuospatial information so as to reason out logical inferences based on the given information. This definition focuses on three aspects: First, it is ability so it varies with age like other cognitive abilities. Second, it requires manipulation of visuospatial information (information about the static and dynamic spatial properties of the object like orientation, degree of rotation, part-whole relation, relative motion etc.) through imagination. Third, such manipulation is needed in order to reason out logical inference. That is, such logically done manipulation leads to objective and stable predictions.

\footnotetext{
${ }^{1}$ Senior Research Fellow, Psychology Research Unit, Indian Statistical Institute, Kolkata, West Bengal, India

${ }^{2}$ Assistant Professor, Psychology Research Unit, Indian Statistical Institute, Kolkata, West Bengal, India *Responding Author

(c) 2016 I S Datta, D Roy; licensee IJIP. This is an Open Access Research distributed under the terms of the Creative Commons Attribution License (http://creativecommons.org/licenses/by/2.0), which permits unrestricted use, distribution, and reproduction in any Medium, provided the original work is properly cited.
} 
Ability to mentally rotate an object in order to accurately predict its transformed shape is one of the most important aspects of visuospatial ability. Maier (1998) defines mental rotation as the "the ability to rapidly and accurately rotate a two- or three-dimensional figure mentally". Reasoning based on mental rotation ability enables an individual to visualize objects in different orientations. This ability is therefore required to solve geometrical problems, map reading as also in performing various daily activities like spatial navigation.

\section{Prior studies on measuring Mental Rotation ability}

The first experiments performed by Shepherd \& Metzler (1971) to measure mental rotation ability were based on the belief that individuals are often able to recognize rotated three-dimensional objects irrespective of the orientation. The three-dimensional objects were all tube-like structures, made of ten cubes and having arms in three axes i.e. $x, y$ and $z$ (Fig. 1). They found that time taken to mentally rotate a three-dimensional object increases as a function of angular difference i.e. as the degree of rotation increases the time taken to predict the transformed figure also increases. Needless to mention they used a digitalized test format where the subjects are presented with two stimuli at a time, one target stimulus and a possible replica. The subject had to determine whether the second image is the same as the target stimulus, but in a rotated position. As they responded, their reaction time was measured.

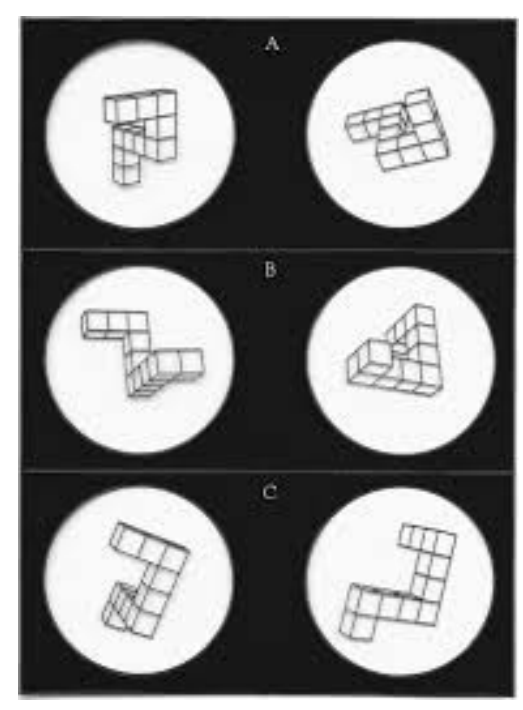

Fig. 1: Stimuli used by Shepard \& Metzler

Later on however, a paper-pencil test of Mental Rotation ability was developed by Vandenberg \& Kuse (1978). This test was used worldwide for a long time until 1995 when a redrawn version of the test was developed by Peters et al., (1995). The redrawn version was then standardized and has been in use worldwide. Here also the target stimuli are the same as that used by Shepard \& Metzler (1971) in their experiments. However, this version is a little different in its stimulus presentation in that, here each target stimulus is presented along with four other options. All the four options are similar looking three-dimensional tube like figures with three arms (Fig. 2). However, only two among these images are that of the target stimulus, although rotated into different orientations. The subject has to identify both the correct images in order to get a score. There are 24 items and the time allotted is 6 minutes.

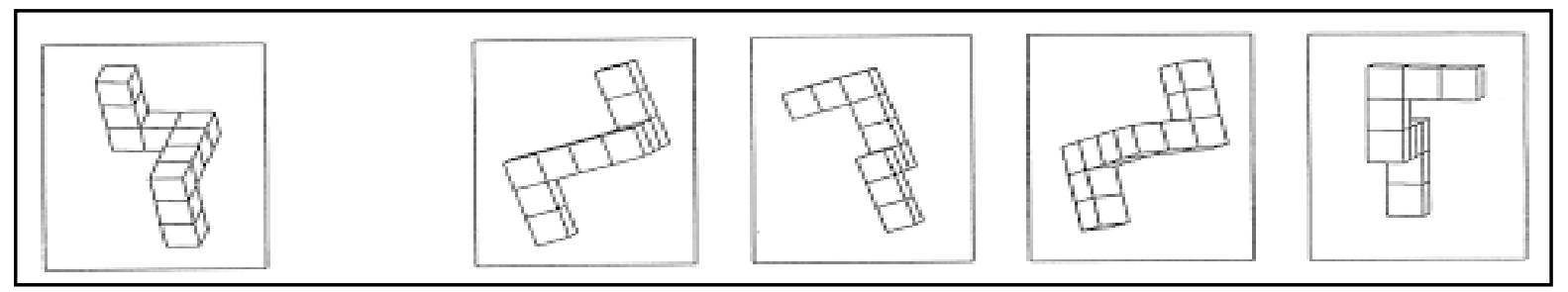

Fig. 2: Item of Redrawn version of Vandenberg \& Kuse Mental Rotation Test (1995) 
As a matter of fact, the task of recognizing two correct options for each item complicates the performance of the subjects. In the original work of Shepard \& Metzler (1971), subjects were asked to compare only one stimulus with the target stimulus at a time. They noticed increase in time taken to respond with increase in the degree of rotation. It is therefore important to consider the outcome when subjects are presented with four rotated images and then to recognize two correct options out of the four. As pointed out by Hoyek et al., (2012), comparing five stimuli at the same time becomes a difficult task and hence several researchers later on reduced the number of stimuli to be compared in their study. This is because the whole process of mental rotation follows three steps: forming a mental representation of the object, performing the rotational transformation and predicting the possible outcome. Now when the subject is presented with a number of options, the subject has to compare each of the presented images with the mentally rotated image. Until he/she finds the correct option, one has to hold the rotated image of the target stimulus in his/her visuospatial working memory. Thus as the number of options to be compared increases, the load on working memory also increases, thereby reducing the chance of answering correctly. Thus the difficulty level of the item increases, not because the subject do not have the ability to mentally rotate the image, but because of greater taxation of working memory space.

Again, since out of four options, the subject has to choose two answers, the guessing probability is $50 \%$ in this case. As the guessing probability increases the accuracy of the test items to adequately discriminate among the respondents will decrease. These issues complicate the administration of the redrawn version of the Vandenberg \& Kuse Mental Rotation Test.

\section{PRESENT STUDY}

There is no denying the fact that mental rotation ability is an important aspect of our spatial reasoning. Therefore it is important to pay attention to its measurement. Present study highlighted the pitfalls of the standard paper-pencil test used for measuring mental rotation ability. Following these, we developed a new test for measuring the same, keeping the stimulus type constant. However, the new test has a different scoring procedure, planned out keeping in mind the drawbacks of the already existing measure of mental rotation ability. Both the tests are then compared based on their average difficulty indices, average performance of the students, internal consistency of items and measure of concurrent validity. Concurrent Validity is measured by correlating the test with a measure of Spatial Visualization, another important Visuospatial Reasoning ability (Lohman, 1979). It is measured by the Space Relations Subtest of the Differential Aptitude Test Battery (DAT). If the new measure proves to be of optimum difficulty level as well as internally more consistent and valid than the standard measure, then it can be concluded that the new test is relatively advantageous than VMRT.

\section{Objective of the study}

The objective of the present study is to examine whether the new test with a different scoring system is a better measure of mental rotation ability than the existing standard test. 


\section{METHOD}

\section{Participants}

Total participants were 147 high school students (Boys=55; Girls=92; Mean age=13.10 years; $\mathrm{SD}=1.84$ ). Among them, $65 \%$ were of age range $11-13$ years while $35 \%$ were of age range $14-16$ years.

\section{Instruments}

a. Redrawn version of VMRT: The redrawn version of Vandenberg \& Kuse Mental Rotation Test (Peters et al., 1995) was administered with instructions in English language. This is a standard measure of mental rotation ability. It has 24 items, each item having a target stimulus and four options, out of which two are correct answers. For obtaining a score of 1 , one has to identify both the correct answers. Maximum score that can be obtained is 24. Time allotted to solve all the 24 items is 6 minutes.

b. ISI-MRT: A new measure of Mental Rotation ability was also administered along with the standard measure. Here also the 3D tube-like figures were used. The stimuli were constructed using 3D Wings software (Figure 3). Each figure has ten cubes of equal size, with arms projecting in $\mathrm{x}, \mathrm{y}$ and $\mathrm{z}$ axes. There are total 20 items, each item having a target stimulus and three options, out of which only one is correct answer. A score of 1 can be obtained on identifying the correct option for each item. Maximum score that can be obtained is 20 . Total time allotted to solve 20 problems is 6 minutes.
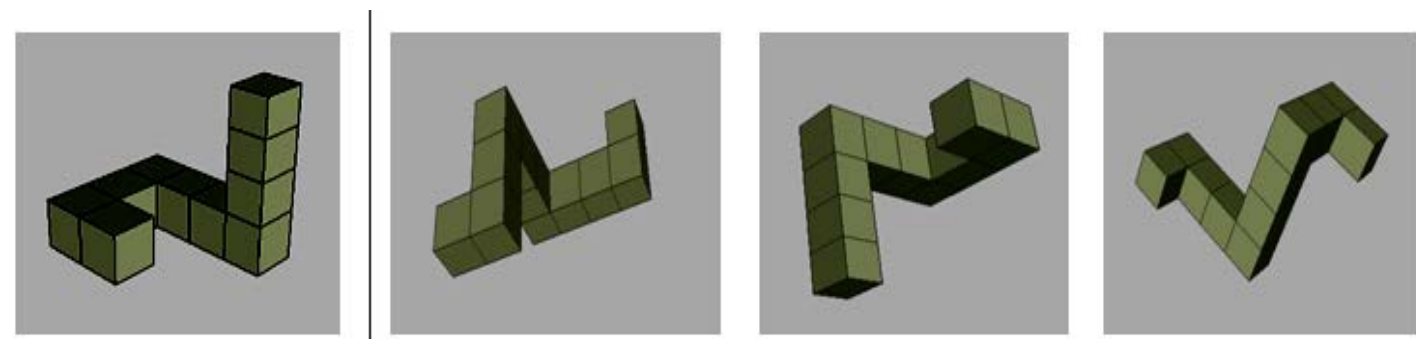

Fig. 3: Item of ISI-MRT test

c. Space Relations subtest of DAT: The Space Relation subtest of Differential Aptitude Test (DAT) (Bennett, Seashore \& Wesman, 1972) was also administered as a measure of Spatial Visualization ability of the students. Spatial visualization is another aspect of Visuospatial Reasoning, and hence a measure of association among the scores of Space Relation subtest and the ISI-MRT will give a measure of its concurrent validity. The Space Relation subtest has 40 items and total time allotted is 25 minutes.

Here, the total score is computed by summating the number of correct responses. The standard formula for calculating the total score as given in the DAT manual has not been followed. This is because here the emphasis is on assessing the ability and not on determining the position of the 
participant in a given population as can be obtained by comparing his/her score with the given norm.

\section{Procedure}

For data collection, permission was sought from local high schools. After obtaining permission, rapport was established with the students. Data were collected in groups separately from junior (grade 7-9) and senior students (grade 10-11). Total time taken for each session was almost 60 minutes. After data collection, participants were provided with small incentives in the form of pens and chocolates. Data were then analysed and results were discussed.

\section{Statistical Analyses}

Scores obtained by the students on both the measures on Mental Rotation ability were compared on the basis of their average difficulty indices and internal consistency. Average performances of the students on both the measures were also compared. Further association among the scores was also examined in comparison to Spatial Visualization scores.

\section{RESULTS \& DISCUSSION}

\section{Comparing Average Difficulty Indices and average performances on both tests}

We found that the average difficulty index of the items of redrawn version of VMRT is very high $(\mathrm{DI}=0.22$ ) while that for ISI-MRT is slightly low (DI=0.67) (Table 1). A test can be considered to be a good measure only if it is able to discriminate among the low and ability respondents. For that it is essential that the test should be of optimum difficulty level. Present findings suggest that the new measure of Mental Rotation ability (ISI-MRT) has average difficulty index lying slightly above the optimum range. While the items of the Redrawn version of Vandenberg \& Kuse Mental Rotation Test (VMRT) are perceived to be very difficult by the adolescents, and hence its average difficulty index dropped down to 0.22 . This is much below the optimum difficulty level ( 0.40 to 0.60$)$ for a test to be a discriminatory one.

High difficulty level of the VMRT is also evident from the average scores obtained by the students on the two tests. While for VMRT, the average score obtained by the students is 5.19 $(S D=2.83)$, for ISI-MRT, it is 13.37 ( $S D=3.31$ ) (Table 1). While dispersion of scores in both the tests is more or less equal, the correct number of responses is much higher in case of ISI-MRT as compared to VMRT. Needless to mention, the students found the VMRT much more difficult to solve as compared to ISI-MRT. 
Table 1. Difficulty Indices and Descriptive Statistics for the two measures of Mental Rotation Ability (N=147)

\begin{tabular}{|c|c|c|c|}
\hline & $\begin{array}{c}\text { Average Difficulty } \\
\text { Index }\end{array}$ & Mean & SD \\
\hline $\begin{array}{c}\text { Redrawn version of Vandenberg \& } \\
\text { Kuse Mental Rotation Test }\end{array}$ & 0.22 & 5.19 & 3.31 \\
\hline ISI-Mental Rotation Test & 0.67 & 13.37 & \\
\hline
\end{tabular}

\section{Comparing Internal consistency of items of both tests}

Perhaps, the high difficulty level of VMRT items rendered the responses to be inconsistent. This is evident from the measure of internal consistency of the two tests, which is found to be much higher in case of ISI-MRT as compared to VMRT. The Kuder-Richardson internal consistency measure for VMRT is only $r=0.57$ while that for ISI-MRT is $r=0.71$ (Table 2). This shows that the items of ISI-MRT are more reliable in that responses to these items are more internally consistent and less random as compared to the items of VMRT. When items are perceived to be difficult, responses become more random and hence inconsistent.

Table 2. Internal Consistency measure of the two tests $(N=147)$.

\begin{tabular}{|c|c|}
\hline $\begin{array}{c}\text { Redrawn version of Vandenberg \& Kuse Mental } \\
\text { Rotation Test }\end{array}$ & 0.57 \\
\hline ISI-Mental Rotation Test & 0.71 \\
\hline
\end{tabular}

\section{Concurrent Validity measure of the two tests}

Further to check for concurrent validity, the association of the two measures with scores obtained in Spatial Relations subtest was checked. While for VMRT, the association is found to be only $r$ $(145)=0.50, \mathrm{p}<0.000$, that for ISI-MRT is found to be $r(145)=0.65, \mathrm{p}<0.000$ (Table 3). The Spatial Relations Subtest is largely used as a measure of Spatial Visualization ability (Kaufman, 2007). Thus it is expected that the mental rotation ability should bear a moderate to high association with spatial visualization ability. This is true in case of ISI-MRT which bears a moderately high correlation with Spatial Relations subtest score, but not for VMRT which shares a low association with the same. 
Table 3. Correlation Matrix for the association among the two measures of Mental Rotation Ability and Spatial Visualization measure ( $N=147)$.

\begin{tabular}{|c|c|c|c|}
\hline & $\mathbf{1}$ & $\mathbf{2}$ & 3 \\
\hline $\begin{array}{c}\text { Redrawn version of } \\
\text { Vandenberg \& Kuse } \\
\text { Mental Rotation Test }\end{array}$ & 1.00 & & \\
\hline ISI-Mental Rotation Test & $0.43^{* *}$ & 1.00 & \\
\hline Spatial Relations subtest & $0.65^{* *}$ & $0.50^{* *}$ & 1.00 \\
\hline
\end{tabular}

${ }^{* *} \mathrm{p}<0.000$

\section{IMPLICATIONS OF THE STUDY}

For a test to be a good measure, it should be of optimum difficulty level. This automatically ensures consistency among the responses and renders it to be a reliable and valid measure. In the present study a more reliable and valid measure of mental rotation ability as compared to a standard measure has been introduced. The newly developed test has an easier scoring system and has lesser items as compared to the standard measure.

Present study also showed how the design and scoring system of a test can make it a more reliable and valid measure or an unreliable and invalid measure altogether. It is therefore always important to consider even the minutest detail while constructing a test.

\section{LIMITATIONS \& CONCLUSION}

However, before considering the findings of the present study it is important to consider the limitations of the study. First, this is a very first attempt to construct a more reliable and valid measure of mental rotation ability in comparison to the standard measure. Further studies therefore, should address the task of standardizing the newly constructed test on a larger population. Second, an initial item analysis of the newly constructed test suggests that the difficulty level is slightly higher than the upper limit of the optimal range. So in further attempts, care should be taken that the average difficulty level lies within the specified optimal range i.e. 40 to 60 . Third, it would be too much to conclude that the respondents perceived the VMRT to be difficult because of its scoring system. There can be actually a number of other reasons. For example, items of ISI-MRT are coloured while those of VMRT are transparent wireframe figures. This might have played a role. Future studies should also look into these issues before making inferences. Lastly, mental rotation ability is a cognitive ability which is largely dependent on time for the perception of the object, formation of a mental representation and finally performing a transformation on it. Faster the ability to transform higher is the ability to mentally rotate an object. Thus only accuracy is not a complete measure of an ability like mental rotation. Needless to mention, no form of paper-pencil test is sufficient enough to accurately tap 
this ability. Thus future studies must consider comparing the standard measure with a computerised measure of mental rotation ability with all the parameters of time taken into account.

In conclusion, it can be said that the newly constructed measure of mental rotation ability has a number of relative advantages than the existing standard measure of the same in a number of ways. However, more in depth research is required to standardize the new test before putting it to use.

\section{REFERENCES}

Bennett, G. K., Seashore, H. G., \& Wesman, A. G. (1972). Form T, Differential Aptitude Tests, Space Relations. New York: Psychological Corporation.

Hoyek, N., Collet, C., Fargier, P., \& Guillot, A. (2012). The use of the Vandenberg and Kuse Mental Rotation Test in children. Journal of Individual Differences, Vol. 33(1):62-67.

Kaufman, S. B. (2007). Sex differences in mental rotation and spatial visualization ability: Can they be accounted for by differences in working memory capacity?. Intelligence, 35(3), 211-223.

Lohman, D. (1979). Spatial Ability: A review and reanalysis of the correlational literature (Tech. Rep. No. 8). Stanford, CA: Stanford University, School of Education.

Maier, P.H. (1998). Spatial geometry and spatial ability: How to make solid geometry solid? In E. Cohors-Fresenborg, K. Reiss, G. Toener and H.-G Weigand (Eds), Selected papers from the Annual Conference of Didactics of Mathematics 1996, Osnabreck, 63-75.

Peters, M., Laeng, B., Latham, K., Jackson, M., Zaiyouna, R., \& Richardson, C. (1995). A redrawn Vandenberg and Kuse mental rotations test-different versions and factors that affect performance. Brain and cognition, 28(1), 39-58.

Shepard, R. N., \& Metzler, J. (1971). Mental rotation of three-dimensional objects.

Thurstone, L. L. (1938). Primary mental abilities. Chicago: University of Chicago Press.

Vandenberg, S. G., \& Kuse, A. R. (1978). Mental rotations, a group test of three-dimensional spatial visualization. Perceptual and motor skills, 47(2), 599-604. 


\section{APPENDIX}

ISI Mental Rotation Test (MRT-ISI)

Instructions: Please look into the figures.
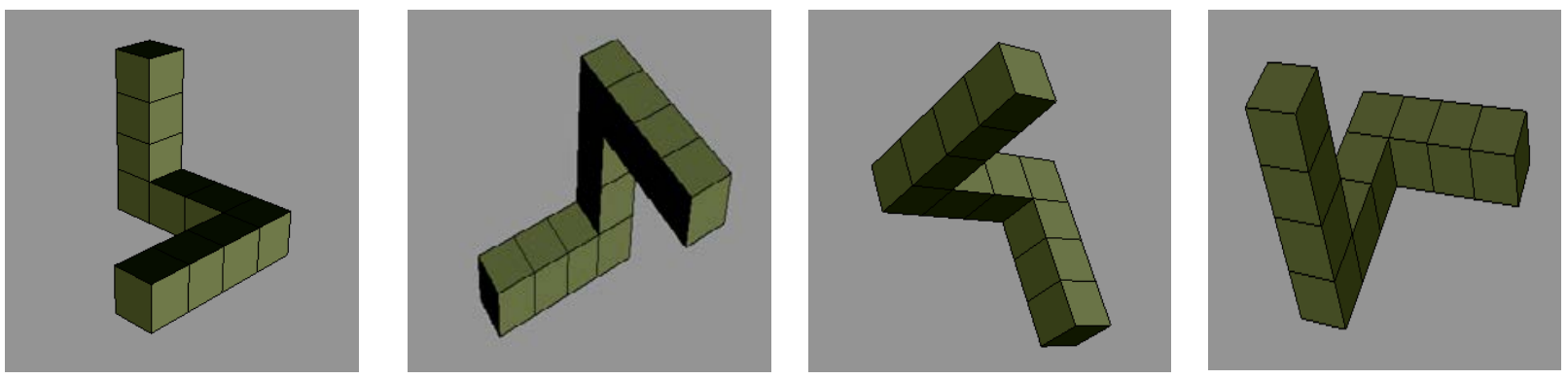

Note that all these pictures are the images of the same object from different viewpoints. The first image if rotated will take the following three positions. Now try to imagine a rotated image of the following figure:

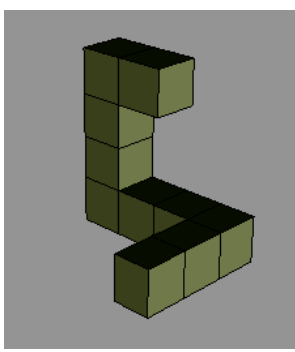

Which among the following pictures is a rotated image of the given image?
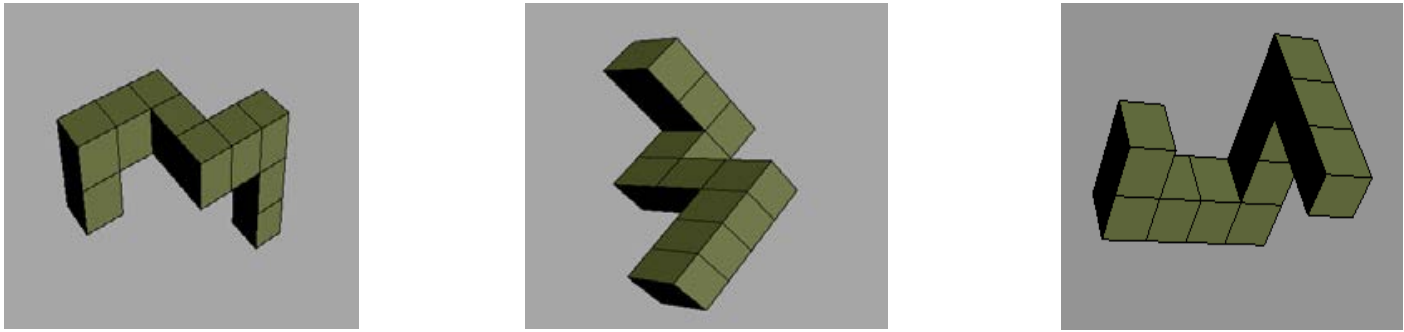

Here the correct answer is the option (c). In the next page you'll find a number of such images with three options for each. You have to identify the correct image out of the three options and indicate it by putting a cross in the circle provided in the answer booklet. 
Construction of Test Measuring Mental Rotation Ability of Adolescent High School Students

1.
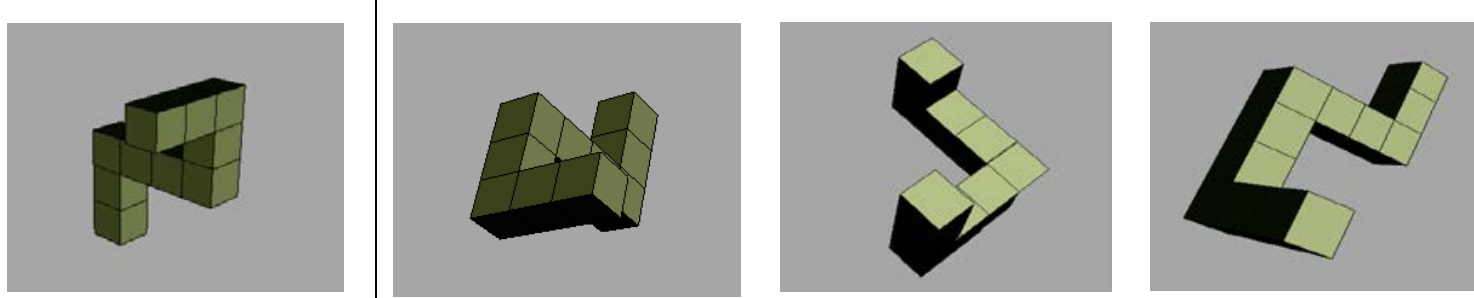

2.
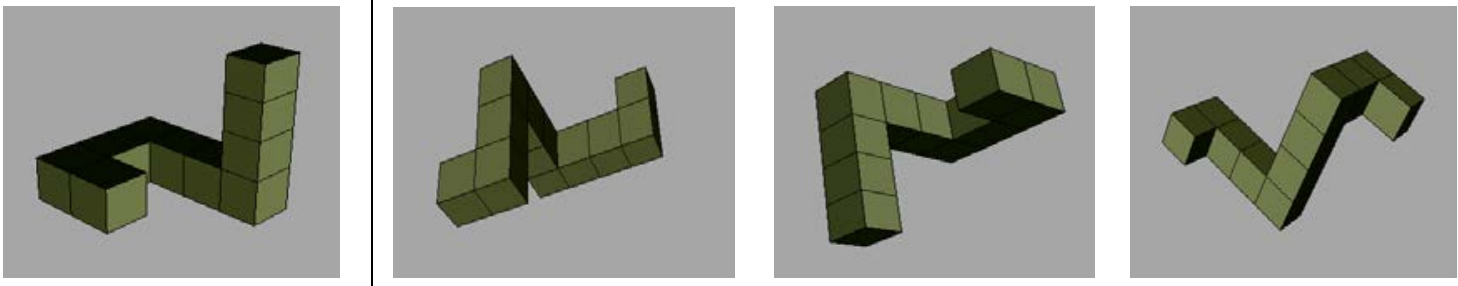

3.
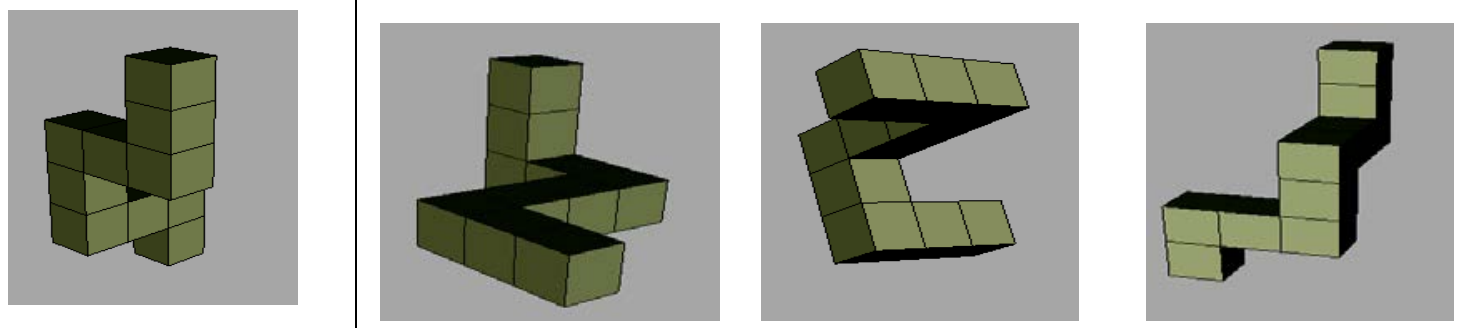